\title{
Breastfeeding may protect children against allergies
}

New results have indicated that atopic disease in children, such as eczema, asthma and food allergies, could be delayed or prevented if children are exclusively breastfed for 4 months or fed instant hydrolyzed formula - lacking cow-milk protein. The new findings from the American Academy of Pediatrics (AAP) replace an earlier policy statement.

The recommendation is also extended to formula-fed infants who are at risk for atopic disease; these are infants with at least one first-degree relative, a parent or sibling, with allergic disease. It is also reported that introduction of solid foods to children's diets appears to have no preventative effect on the acquisition of atopic diseases.

Frank Greer, author of the report and professor of pediatrics at the University of Wisconsin, WI, USA, and chairman of the AAP Committee on Nutrition, explained, "The best prevention for atopic disease is exclu-

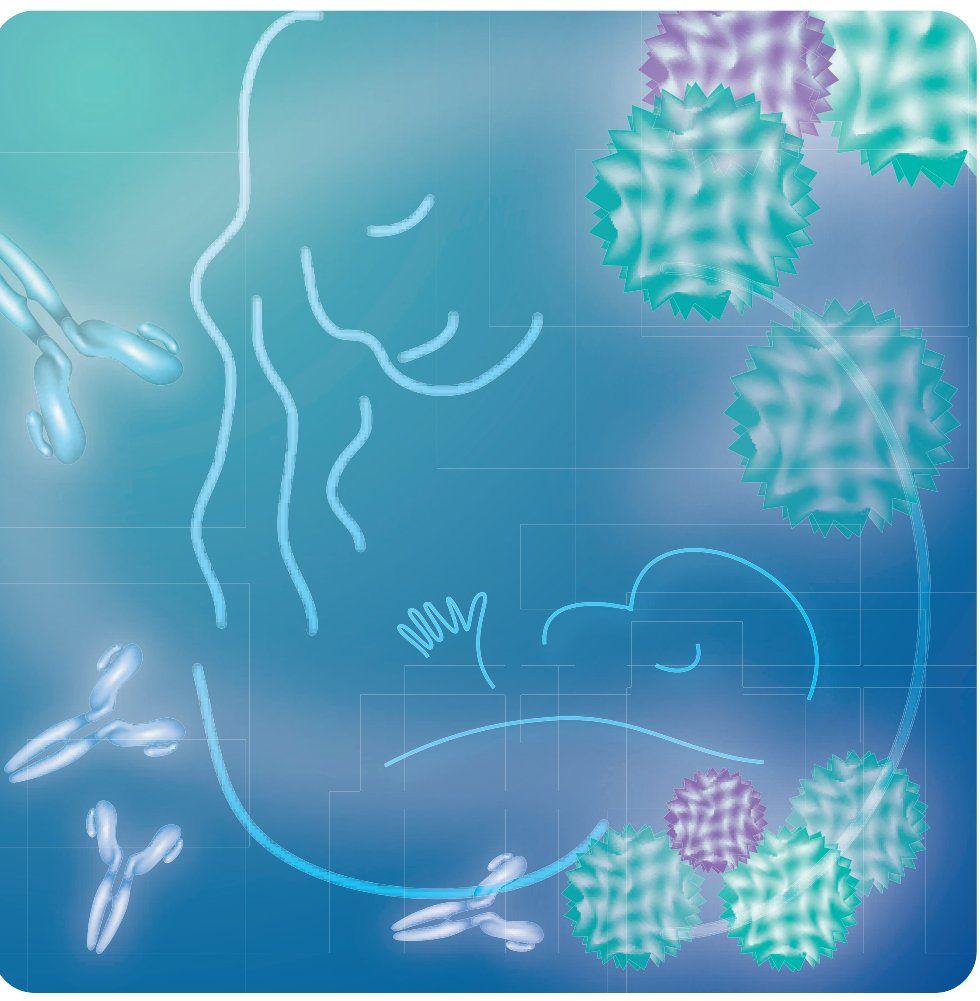

hoped that maternal diet, early-onset of breastfeeding and introduction of early childhood diet may all play important roles in allergy prevention. The incidence of asthma in children of 4 years of age and younger has risen $160 \%$, and the incidence of atopic dermatitis is almost three-times higher. Alarmingly, in the past decade, peanut allergy in young children has also doubled.

Results from the new report concluded that there is currently no evidence to support the notion that what a mother eats during pregnancy or breastfeeding plays a major role in preventing atopic disease in infants. However, it has been proven that avoiding certain foods during breastfeeding may help prevent atopic eczema. Exclusive breastfeeding for at least 4 months in infants at a high-risk of developing atopic disease has also been demonstrated to decrease the risk of developing eczema and cowmilk allergy during the first 2 years of life.

The use of hydrolyzed infant formula, which does not contain cow-milk protein, could also delay or prevent atopic dermatitis in high-risk infants who are not exclusively fed on breast milk for 4 months; not all formulas have the same protective benefit. Infants can be protected against wheezing in early life if exclusively breastfed for 3 months.

Currently there is no supporting evidence to suggest that any type of dietary intervention will

Current studies have indicated that only breastfeeding has a significant impact on atopic dermatitis, with a decrease of approximately a third.

It is well-known that genetic factors play an important role in the development of these diseases and allergies, but it is now thought that the impact of environmental factors has been underestimated. prevent atopic disease after the child is 4 months of age. The study requires further long-term follow-up in children. Source: Greer FR, Sicherer SH, Burks AW: Effects of early nutritional interventions on the development of atopic disease in infants and children: the role of maternal dietary restriction, breastfeeding, timing of introduction of complementary foods, and hydrolyzed formulas. Pediatrics 121(1), 183-191 (2008). dence of asthma, eczema and food allergies over the last few decades, it was 


\section{in brief..}

Adolescent overweight and future adult coronary heart disease.

Bibbins-Domingo K, Coxson P, Pletcher MJ, Lightwood J, Goldman L. N. Engl. J. Med. 6, 357(23), 2325-2327 (2007).

Predicts the effect of adolescent overweight on future adult coronary heart disease (CHD). The prevalence of obese 35 year olds in 2020 was estimated on the basis of the patient's weight in 2000. The CHD Policy Mode was utilized to project the annual excess incidence and prevalence of CHD. It was predicted that adolescent overweight will increase the prevalence of obese 35 year olds in 2020 to $30-37 \%$ in men and $34-44 \%$ in women. As a consequence of this increased obesity, an increase in the incidence of CHD and in the total number of CHD events and deaths is projected to occur in young adulthood, and it is predicted that by 2035 the prevalence of CHD will increase by a range of 5 to $16 \%$.

Growth hormone treatment of early growth failure in toddlers with Turner syndrome: a randomized, controlled, multicenter trial.

Davenport ML, Crowe BJ, Travers SH et al.: J. Clin. Endocrinol. Metab. 92(9), 3406-3416 (2008).

Determines whether growth hormone $(\mathrm{GH})$ treatments initiated before 4 years of age in girls with Turner syndrome (TS) can prevent growth failure. A prospective, randomized, controlled, openlabel, multicenter clinical study was conducted on 88 girls aged 9 months to 4 years of age. SD scores were used to calculate the difference in height between the treated and control group. The mean height of patients increased in the GH group. No new or unexpected side effects associated with $\mathrm{GH}$ treatment were detected and it was concluded that early GH treatment can correct growth failure and normalize height in infants and toddlers with TS.

Automated peritoneal dialysis as the modality of choice: a single-center, 3-year experience with 458 children in Mexico. Fabian Velasco R, Lagunas Muñoz J,

Sanchez Saavedra V et al.: Pediatr. Nephrol. (2007) (Epub ahead of print).

Discusses the use of automated peritoneal dialysis (APD) as the best dialysis modality for pediatric patients. The study reports a 3-year ADP experience, involving 458 end-stage renal disease (ERSD) patients. Patients who were being treated with continuous ambulatory peritoneal dialysis (CAPD) were switched to APD. Improvements in daily ultrafiltration were observed after the switch. The presence of edema decreased from 67 to $8 \%$, patients requiring antihypertensive drugs also dropped. The peritonitis rate improved from one episode every 35 patients/month to one episode every 47 patients/month and overall hospitalization decreased.

\section{Pediatric cancer patients exhibit significantly higher prevalence of morphologic abnormalities}

Pediatric cancer patients have a significantly higher prevalence of morphologic abnormalities than controls. Children with cancer were shown to have a higher incidence of both single and combined morphologic abnormalities, suggesting that constitutional genetic defects may play a more important role in pediatric cancers than has been estimated.

The study enrolled 1073 pediatric cancer patients; all patients underwent a physical examination that was directed at 683 morphologic abnormalities; 1007 healthy children served as controls and were examined identically.

Results indicated that major and minor abnormalities were significantly more prevalent among patients with cancer than among controls. A total of 268 major abnormalities per 1000 cases were observed in the pediatric cancer group, versus 155 in the controls.

"In $3.9 \%$ of pediatric cancer patients, an established clinical genetic syndrome is diagnosed, which is much higher than in the general population," explained lead author Johannes Merks from the Emma Children's Hospital, Academic Medical Center, Amsterdam, The Netherlands.

"Half of the syndromes were missed despite intensive general pediatric care, indicating that all childhood cancer patients deserve an evaluation by a clinical geneticist or pediatrician trained in clinical morphology," Merks added.

Merks explained that they now have to confirm the two new tumor predisposition syndromes in an independent cohort. It is speculated that once the genetic defects have been identified, they could be used for early diagnosis and treatment of the syndrome.

Source: Merks JH, Ozgen HM, Koster J, Zwinderman AH, Caron HN, Hennekam RC: Prevalence and patterns of morphological abnormalities in patients with childhood cancer. JAMA 299(1), 61-69 (2008).

\section{AAP releases 2008 immunization schedules for children and adolescents}

The recommended immunization schedules for children and adolescents in the USA for 2008 have been released by The American Academy of Pediatrics (AAP).

Recommendations include; a single dose of pneumoccoal conjugate vaccine for healthy children aged 24-59 months who are incompletely immunized; expansion of the age recommendations for the use of the live-attenuated influenza vaccine to include healthy children as young as 2-years old; a reduced time interval for the administration of the second dose of live-attenuated nasal influenza vaccine (when a second dose is required) from 6 to 4 weeks; and a single dose of quadrivalent meningococcal conjugate vaccine for all adolescents 11-18 years of age, if not previously administered. These recommendations reflect information supplied by the US FDA, for the use of FDA-licensed vaccines.

Source: The American Academy of Pediatrics: Recommended Immunization Schedules for Children and Adolescents United States, 2008. www.aapredbook. org/news/vaccstatus.shtml 


\section{Studies suggest that home treatment of severe pneumonia in children is safe and effective}

The Lancet has revealed that home treatment of severe pneumonia in children with oral antibiotics is as safe and effective as treatment in hospital. These findings have motivated the WHO to update and revise the current recommendations on the treatment of severe pneumonia in children.

Current guidelines recommend that children with fast breathing (nonsevere pneumonia) be treated at home with oral antibiotics, and those with lower-chest indrawing, severe pneumonia and general danger signs and very severe disease, be referred to hospital and treated with parenteral antibiotics, benzylpenicilllin or ampicillin. A concern is that children in developing countries do not always have access to hospitals, so safe community-based treatments are a preferred alternative that would reduce cost and in-hospital hazards.
The team, led by Donal Thea, Boston University School of Public Health, Boston, USA, studied 2037 children between the ages of 3 and 59 months, over seven study sites located in Pakistan, to determine whether home-based treatment is as safe and effective as in-hospital treatment.

The trail was split into two groups, children were selected at random to receive oral amoxicillin syrup and were directed to go home. The other trial group received intravenous ampicillin for $48 \mathrm{~h}$ as an inpatient.

A total of four children from the hospitalized group, and one child from the home group died due to treatment failure. None of the deaths were considered to be associated with treatment allocation and no serious adverse events were reported.

The authors concluded that, "Home treatment with high-dose oral amoxicillin is equivalent to currently recommended hospitalization and parenteral ampicillin for treatment of severe pneumonia without underlying complications, suggesting that WHO recommendations for treatment of severe pneumonia need to be revised."

"Because the agents that cause pneumonia in Pakistani children do not differ from those in most other developing countries, our findings have important public-health policy and programmatic implications, particularly for Integrated Management of Childhood Illness (IMCI) guidelines ... ambulatory management of severe pneumonia in children has the potential to improve access to care and inequity while reducing costs and mortality from pneumonia."

Source: Hazir T, Fox LM, Nisar YB et al.: Ambulatory short-course high-dose oral amoxicillin for treatment of severe pneumonia in children: a randomised equivalency trial. Lancet 371(9606), 49-56 (2008).

\section{No link between hepatitis B vaccine and childhood multiple sclerosis}

The Archives of Pediatrics \& Adolescent Medicine has stated that vaccinating against the hepatitis B virus does not appear to be associated with the risk of developing multiple sclerosis (MS) in childhood.

Numerous studies have evaluated the association between the vaccine and the development of MS in adults, no significant increase in the risk of MS in the short or long-term have been found.

The results are based on the study of 143 children who developed MS before 16 years of age, with a first episode of the disease occurring between 1994 and 2003. Each patient was matched to an average of eight control participants from the general population. The control patents all match in sex, age and location, but did not have MS. Telephone interviews and questionnaires were used to collect vaccination records and information about family history of MS or other autoimmune diseases.

Results obtained showed that in the 3 years before the first episode of MS, approximately $32 \%$ of both the MS and control groups vaccinated against hepatitis B.

Lead author Yann Mikaeloff claimed that, "Vaccination against hepatitis B within the 3-year study period was not associated with an increased rate of a first episode of MS," adding that, "the rate was also not increased for hepatitis B vaccination within 6 months of the index date or at any time since birth or as a function of the number of injections or the brand of hepatitis B vaccine."
Mikaeloff, also speculated that, "some of these epidemiologic studies have been criticized for methodological limitations," and added, "this controversy created public misgivings about hepatitis $\mathrm{B}$ vaccination. Hepatitis $\mathrm{B}$ vaccination in children remained low in several countries despite vaccination campaigns supporting early vaccination against hepatitis $\mathrm{B}$ in children as a means of inducing strong and longlasting immunity and despite high levels of hepatitis B-related morbidity and mortality worldwide."

Source: Mikaeloff Y, Caridade G, Rossier M, Suissa S, Tardieu M: Hepatitis B vaccination and the risk of childhood-onset multiple sclerosis. Arch. Pediatr. Adolesc. Med. 161(12), 1176-1182 (2008). 